\title{
Effect of Different Solvents on Biginelli Reaction Using Layered Double Hyroxide (LDH) As an Ecofriendly Catalyst
}

\author{
N. S. Ghotkar, D. A. Pund, B. N. Berad \\ ${ }^{1}$ Dr. Sau. Kamaltai Gawai Institute of Engineering and Technology, Darapur, Dist. Amravati. \\ Maharashtra. India \\ ${ }^{2}$ Shri R. R. Lahoti Science College Morshi. Amravati. Maharashtra. India \\ ${ }^{3}$ Shri Shivaji Science College Amravati, Maharashtra. India
}

\begin{abstract}
Article Info

Volume 8, Issue 1

Page Number: 223-226

Publication Issue :

January-February-2021

Article History

Accepted : 08 Feb 2021

Published : 18 Feb 2021

A simple and efficient method of the 3,4- dihydropyrimidin-2(1H)ones/thiones synthesis in good to high yield is described. It consists in a onepot three-component Biginelli condensation using Layered double hydroxide (LDH) as catalyst. The effect of different solvents on the yields of final product also been studied. Highly polar solvent as well as highly non-polar gives less yields in presence of LDH catalyst.

Keywords :- Biginelli reaction, one-pot procedure, 3,4-dihydropyrimidin2(1H)- ones, LDH.
\end{abstract}

\section{INTRODUCTION}

Multicomponts reactions in which three or more reactants are combined in a single vessel to generate new molecules that contain portions of each reactant undoubtedly maintain great importance in organic and medicinal chemistry due to synthetic efficiency and molecular diversity required in discovery of new compounds (1). The Biginelli reaction (2) is one of the most useful multicomponts reactions. The Most simple and straight forward procedure, involves one-pot condensation of ethyl acetoacetate, benzaldehyde and urea under strongly acidic conditions at elevated temperature (3). However one serious drawback of Biginelli reaction is low yields in case of substituted aromatic and aliphatic aldehydes.
This has led to the developments of multistep strategies that produce somewhat higher overall yields but lack of simplicity of the multicompoments synthesis (4). Thus during the last two decades Biginelli reaction for the synthesis of dihydropyriminines has received renewed interest and several improved procedures has been reported (5) including, in particular the use, as catalysts, of Lewis acids such as Indium (III) chloride (6), Indium(III) Bromide (7), $\mathrm{CuCl}_{2} / \mathrm{CuSO}_{4}$ or $\mathrm{CuCl}_{2} / \mathrm{HCl}$ (8), Strontium (II) triflate (9) , Bismuth (III) nitrate (10), Calcium chloride (11), Cupric (II) triflate (12), sulphate Zirconia (13), $\mathrm{Co}\left(\mathrm{HSO}_{4}\right)_{2}$ (14), Chlorotrimethyl silane (15) , copper (II) acetate / sodium ascorbate $\mathrm{AcOH}$ (16), Iron (III) tosylate(17). Biginelli reaction was also catalyzed by bases such as $\left(\mathrm{NH}_{4}\right)_{2} \mathrm{CO}_{3} \quad(18)$, t- 
$\mathrm{BuOK}$ (19) and by organocatalysts as for examples L-proline (20) phenyl phosphoric acid (21) and primary amine (22). Nanoparticules (23) , hetropoly acids (24), and ionic liquids (25) have been successfully applied to the Biginelli products synthesis, In addition, a significant improvement was observed in the dihydrpyrimidine synthesis under ultrasonic (26) and microwave irradiation (27) Furthermore these are only few enantioselective methods synthesis (28) of 3,4-dihydropyrimidin-2(1H)-one. Yet many methods require harsh conditions, expensive or dangerous catalysts, long reaction time and the use of non-ecofriendly solvents consequently, the search of simple, efficient, environmentally benign, inexpensive with better yields procedures remains valid.

We report here our preliminary investigation dealing with the use of Layered double Hydroxide is a simple, easy to prepare and ecofriendly catalyst in Biginelli reaction.

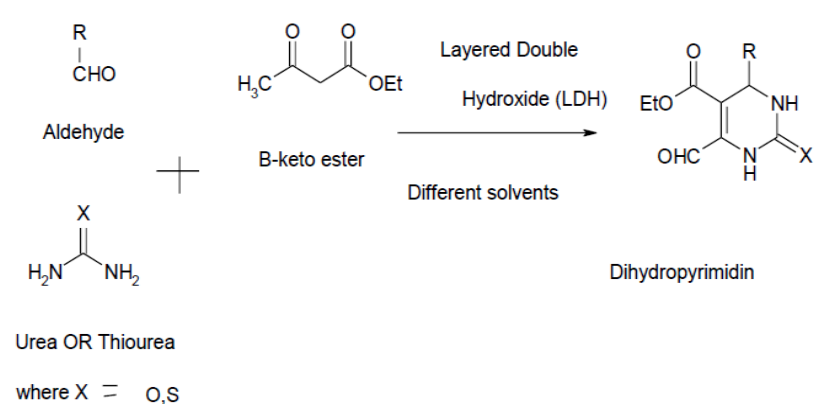

\section{RESULTS AND DISCUSSION}

The optical reaction conditions for the multicomponent Biginelli reaction were established after several attempts to obtain the products using different solvents, catalysts amounts, and temperatures. Initially, we investigated the Biginelli condensation of benzaldehyde (1 equiv), ethyl acetoacetate) lequive), and urea (1.5 eqimolar) catalysed by 2 grms of layered double hydroxide. We were pleased to find that the catalyst provided good yield of the corresponding DHPM.

Next the amount of the catalyst was evaluated and the results are shown in Table 1 . In terms of yield 2 moles of LDH gave the best results (Entry 4). The use of $0.5 \mathrm{gm}$ to $1.5 \mathrm{gm}$ of the catalyst caused a slight increase in the yield (Entry 1-3). In addition raising the catalyst load from 2.5 to $3.0 \mathrm{gm}$ led to constant reaction yields (Entry 5 to 6$)$.

\section{Table No 1}

\begin{tabular}{|c|c|c|c|c|c|}
\hline $\begin{array}{l}\text { Ent } \\
\text { ry }\end{array}$ & $\begin{array}{l}\text { Solv } \\
\text { e nt }\end{array}$ & $\begin{array}{l}\text { Cata } \\
1 \text { yst }\end{array}$ & $\begin{array}{l}\text { Tim } \\
\mathrm{e}\end{array}$ & $\begin{array}{l}\text { Tempe } \\
\text { rature }\end{array}$ & $\begin{array}{c}\text { Yie } \\
\text { ld } \\
\%\end{array}$ \\
\hline 1 & $\begin{array}{c}\text { Etha } \\
\text { nol }\end{array}$ & $\begin{array}{l}0.5 \\
\mathrm{gm}\end{array}$ & $4 \mathrm{hrs}$ & Reflux & 82 \\
\hline 2 & $\begin{array}{c}\text { Etha } \\
\text { nol }\end{array}$ & $\begin{array}{l}1.0 \\
\mathrm{gm}\end{array}$ & $4 \mathrm{hrs}$ & Reflux & 84 \\
\hline 3 & $\begin{array}{l}\text { Etha } \\
\text { nol }\end{array}$ & $\begin{array}{l}1.5 \\
\mathrm{gm}\end{array}$ & $4 \mathrm{hrs}$ & Reflux & 86 \\
\hline 4 & $\begin{array}{c}\text { Etha } \\
\text { nol }\end{array}$ & $\begin{array}{l}2.0 \\
\mathrm{gm}\end{array}$ & $4 \mathrm{hrs}$ & Reflux & 88 \\
\hline 5 & $\begin{array}{c}\text { Etha } \\
\text { nol }\end{array}$ & $\begin{array}{l}2.5 \\
\mathrm{gm}\end{array}$ & $4 \mathrm{hrs}$ & Reflux & 88 \\
\hline 6 & $\begin{array}{l}\text { Etha } \\
\text { nol }\end{array}$ & $\begin{array}{l}3.0 \\
\mathrm{gm}\end{array}$ & $4 \mathrm{hrs}$ & Reflux & 88 \\
\hline
\end{tabular}

A further survey of solvents using $0.5 \mathrm{gm}$ of LDH revealed that ethanol solvent was the most favorable shown in table 2, common solvents such as water (entry 5), and THF (Entry 3), led to poorer yields, and only traces of products was observed. Also lower yields were obtained with toluene (Entry 4) and Ethylene Glycol (Entry 7). When ethanol (Entry 2) and CHCI3 (Entry 1) were used as solvents, the obtained yields were slightly elevated to $70 \%$ and $82 \%$ respectively. The reaction is also slower in all other solvents (Entries 1-7). 
Table No 2

\begin{tabular}{|c|l|c|l|c|c|}
\hline $\begin{array}{c}\text { E } \\
\text { nt } \\
\text { ry }\end{array}$ & Solvent & $\begin{array}{c}\text { Catal } \\
\text { yst }\end{array}$ & $\begin{array}{c}\text { Tempera } \\
\text { ture }\end{array}$ & $\begin{array}{c}\text { Ti } \\
\text { me }\end{array}$ & $\begin{array}{c}\text { Yie } \\
\text { ld }\end{array}$ \\
\hline 1 & CHCI3 & $\begin{array}{c}0.5 \mathrm{~g} \\
\mathrm{~m}\end{array}$ & Reflux & $\begin{array}{c}4 \mathrm{hr} \\
\mathrm{s}\end{array}$ & 70 \\
\hline 2 & Ethanol & $\begin{array}{c}0.5 \mathrm{~g} \\
\mathrm{~m}\end{array}$ & Reflux & $\begin{array}{c}4 \mathrm{hr} \\
\mathrm{s}\end{array}$ & 82 \\
\hline 3 & THF & $\begin{array}{c}0.5 \mathrm{~g} \\
\mathrm{~m}\end{array}$ & Reflux & $\begin{array}{c}4 \mathrm{hr} \\
\mathrm{s}\end{array}$ & $\begin{array}{c}\text { Tra } \\
\mathrm{ce}\end{array}$ \\
\hline 4 & Tolune & $\begin{array}{c}0.5 \mathrm{~g} \\
\mathrm{~m}\end{array}$ & Reflux & $\begin{array}{c}4 \mathrm{hr} \\
\mathrm{s}\end{array}$ & 43 \\
\hline 5 & Water & $\begin{array}{c}0.5 \mathrm{~g} \\
\mathrm{~m}\end{array}$ & Reflux & $\begin{array}{c}4 \mathrm{hr} \\
\mathrm{s}\end{array}$ & $\begin{array}{c}\text { Tra } \\
\mathrm{ce}\end{array}$ \\
\hline 6 & CH3C & $\begin{array}{c}0.5 \mathrm{~g} \\
\mathrm{~m}\end{array}$ & Reflux & $\begin{array}{c}4 \mathrm{hr} \\
\mathrm{s}\end{array}$ & 40 \\
\hline 7 & $\begin{array}{c}\text { Ethylen e } \\
\text { Glycol }\end{array}$ & $\begin{array}{c}0.5 \mathrm{~g} \\
\mathrm{~m}\end{array}$ & Reflux & $\begin{array}{c}4 \mathrm{hr} \\
\mathrm{s}\end{array}$ & 58 \\
& & & & \\
\hline
\end{tabular}

\section{III.EXPERMENTAL SECTION}

Melting point were measured using a fine control Electro thermal capillary apparatus and are uncorrected. IR spectra were recorded from $\mathrm{KBr}$ disk on a Shimadzu FT-IR 8201 PC spectrometer. The $1 \mathrm{H}$ and $13 \mathrm{C}$ NMR spectra were obtained with a Burker $250 \mathrm{MHz}$ spectrometer in DMSO.

General synthesis for the synthesis of 3,4dihydropyrimidin-2(1H)-ones. A mixture of aldehyde $1(1.0 \mathrm{~m} \mathrm{~mol})$, the appropriate $\mathrm{B}$ ketoester or 1,3-diketone $2(1.0 \mathrm{~m} \mathrm{~mol})$ urea or thiourea 3 ( $1.5 \mathrm{~m} \mathrm{~mol}$ ), and LDH ( $2.0 \mathrm{gm}$ ) was heated under ethanol solvent at $78 \mathrm{C}$ for the appropriate time indicated in table 2. After being cooled, the reaction mixture was then poured onto crused ice, and steered for 5-10 minutes. The predicated product was filtered under suction, washed with cold water. Analytically pure sample were prepared by recrystlisation from ethanol.

Ethyl-4-(Phenyl)-6-methyl-2oxo-1,2,3,4tetrahydro-5-pyrimidine carboxylate :-
Status:- Solid. M.P. - 203C Reported 202- 204C, IR: 3171(-NH-), 3114(-NH-), 1651(C=O),1670(C=O), 2901(-C-H), 2937, 2984(-C-H), 1252,1282 (C-O) 1H NMR: (3.25 d 3H), (3.17, t 2H), (4.2,q, 2H), (5.3,S,2H), (7.2-7.4 Ar-CH, 5H).

\section{IV.CONCLUSION}

In conclusion we have developed an environmentally friendly method for the synthesis of 3,4 dihydropyrimidiones by using LDH ( Layered Double Hydroxide) as an inexpensive and easily available catalyst using different solvent. In addition to its simplicity and mild reaction condition, it tolerate a wide variety of substitutions in all three components. The adopted procedure is convenient, involves simple experimental procedure and product isolation, therefore, it is a useful addition to the existing methods.

\section{ACKNOWLEDGEMENT}

The authors are thankful to the Director, Government Viderbha Science and humanities, Amravati for providing necessary facilities for the research work.

\section{REFERENCES}

[1]. Multicomponents reactions, $\mathrm{Zhu}, \mathrm{J}$, bienayme, $\mathrm{H}$. Eds, Wiley-VCH: weinheim, Germaney 2005.

[2]. P- Biginelli, Gazz. Chim. Ital. 1893, 23, 360-416.

[3]. CO Kappe,Tetrahedron1993,49,6937- 6963.

[4]. A) j barluenga, m Thomas, A Ballesteros, L A lopaz, Tetrahedron Letters 1989, 30, 4573- 4576. B) A D Shutalev, EA Kishko, N Sinova , AY Kuznetsov, Molecules 1998, 3, 100-106.

[5]. S Sandhu, JS Sandhu, Arkivoc 2012, I , 66-133.

[6]. BC Ranu , A Hajara , U Jan, J. Org. Chem. 2000, 65, 6270-6272.

[7]. NY fu, YF Yuan, SW Wang, JT Wang, C peppe, Tetrahedron 2002, 588,4801-4807. 
[8]. a) M Gohain , D Prajapati, JS Sandhu, Synlett 2004, 2, 235-238. b) S jaykumar, TK Sabeer, J Chem. Pharma. Res.2011, 3, 1089-1096.

[9]. W Su, J Li, Z Zheng, Y Shen, Tetrahedron Lett. 2005, 45,6037-6040.

[10].M M khodei, A R Khosropour, M Jowkar, Synthesis 2005, 8, 1301.

[11]. B Gangadasu, P Narender, B C Raju, V J Rao, Indian J. Chem. 2006,45, 1259.

[12]. A S Paraskar, G K Dzwkar, A Sudalai, Tetrahedron Lett. 2003, 3305-3308.

[13]. D Angeles-Bentran, 1 lomas- Romero, V H laraCorona, E Gonzales- Zamora, g Negron- Silva, Molecoles 2006, 11,731.

[14].HR memarian, m Ranjbar, J. Chin. Chem. Soc. 2011, 58, 522-527.

[15].S Khodadoost, E Alipoor, M Amini, Org. Chem. J 2010,2, 85-88.

[16].P Salehi, m Dbiri, m Khooshari, SK Movahed, M Bararjanian, Mol. Divers 2011, 15, 833.

[17].JT Starcevitch, TJ Laughlin, RS Mohan , Tetraheron Lett. 2013,54,983- 985.

[18].F Tamaddon, Z Ramzzi , AA Jafari, Tetrahedron Lett.2010, 51, 1187-1189.

[19].ZL Shen , XP Xu, SJ ji, J. Org. Chem. 2010, 75(4), 1162-1167.

[20]. JS Yadao, SP Kumar, G Kondaji, R Shrinivasa Rao, K Nagaiah, Chem. Lett. 2004,33, 1168.

[21]. A D Sagar, S m Reddy, JS Pulle, M V Yadao, J. Chem. Phar. Res. 2011,3,649-654.

[22]. D Ding, C G Zhao, Europian J. Org. Chem.2010, 20, 3802-3805.

[23].a) SB Sapcal, KF Shelke, B B Shingate, M S Shingate, Bull. Koriean Chem.Soc.2010,31, 351-354.

b) m Nasr- Esfahani, SJ Hoseini, F Mohammadi, Chinease J Cata. 2011, 32, 1484-1489. C0) RJ Kalbashi, AR Massah, B Daneshvarnejad, Appl. Clay Sc.2012, 55, 1-9. D) E Kovari, N Koukabasi,O Armandpour, Tetrahedron 2014, 70, 1383-1386.

[24].a ) E Rafiee H Jafari, Bioorg. Med. Chem. Lett. 2006, 16, 2463-2455. B) M Mossavifer C.R. Chim 2012, 15, 444- 447.c) M M Herari, K Bakhtiari, Catal. Commun. 2006,7, 373- 376.
[25].A) H Kefayati, F Asghari, R Khanjanian, J. Mol. Liq. 2012, 172, 147-151. B) J Peng Y Deng, Tetrahedron Lett. 2001, 42, 5917-5919. C) HGO Alvim , I B de Lima, HCB deoliveira, FC Gozzo, JL de Macedo, PV Abdelnur, WA Siliva, BAD Neto, ACS Catal. 2013,3, 1442-1430.

[26].H A Stefani, C B Oliveira, R B Almeida, CMP Pereira , R C Braga, R Cella, V C Borges, L Savegnano, CW Nogueira, Eur. J. Med. Chem. 2006, 41, 513-518. B) S Singhal, J K Joseph, S L Jain, B Sain, Green Chem. Lett. Rev. 2010, 3, 23-26. C) AMA Al-Kadassi, G M Nazeruddin, J. Chem. Pharma. Res. 2010, 2, 536-543.

[27].B K Banik, TR Anupama, A Datta, C Mukhopadhyay, Terahedron Lett. 2007, 48, 73927394. B) KK Pasunooti, H Chai, CN Jensen, BK Gorityala, S Wang, XM Liu, Tetrahedron Lett. 2011, 52, 80-84.

[28].A) JH Sohn, HM Choi, S Lee, S Joung HY Lee, Eur. J . Org. Chem. 2009, 23, 3858-3862. B) J P Wan , Y J Pan, Chem. Commun. 2009, 19, 2768-2770. C) M A Blasco, S Thumann J Wittmann, A Giannis, H Gorger, Bioorg. Med. Chem. Lett. 2010, 20. 46794682 .

\section{Cite this article as :}

N. S. Ghotkar, D. A. Pund, B. N. Berad, "Effect of Different Solvents on Biginelli Reaction Using Layered Double Hyroxide (LDH) As an Ecofriendly Catalyst", International Journal of Scientific Research in Science and Technology (IJSRST), Online ISSN : 2395-602X, Print ISSN : 2395-6011, Volume 8 Issue 1, pp. 223-226, January-February 2021. Available at doi : https://doi.org/10.32628/IJSRST218128 Journal URL : https://ijsrst.com/IJSRST218128 